\title{
The hyperflexed seemingly useless tetraplegic hand: a method of surgical amelioration
}

\author{
W J Treanor MD FACP, ${ }^{1}$ E Moberg MD PhD,${ }^{2} \mathrm{H}$ J Buncke MD FACS ${ }^{3}$ \\ ${ }^{1}$ North Coast Rehabilitation Center, Santa Rosa, California, USA; ${ }^{2}$ Sahlgren University \\ Hospital, Goteberg, Sweden; ${ }^{3}$ Microsurgical Replantation Transplantation Service, Davies \\ Medical Center, San Francisco, California, USA.
}

In a series of 65 tetraplegic hands with severe disabling spasticity and/or flexion contracture, selective flexor tendon elongation procedures were employed to improve their static and kinetic postures. A kinetic approach was adopted, utilizing local anesthesia in a wide-awake patient. This was employed to allow for the patient's cooperation in determining at surgery the desired length of digital extension at the time of wrist tenodesis action. Elongation of the extensor digitorum communis (EDC), extensor indicis proprius (EIP), and extensor digiti quinti (ED V) were added to correct an 'extrinsic-plus' posture observed in 16 patients following flexor tendon lengthening. With the resultant improvement in the static posture of the digits, tendon transfers could then be employed to provide a more functional tenodesis action. These measures provided both improved palmar contact and prehension.

Key words: tetraplegia; tetraplegic hand spasticity; contracture; surgery.

\section{Introduction}

'In the Museum of the Royal College of Surgeons of London there is a specimen of the upper part of the spinal cord, laid open to show a healed fracture of the lower part of the neck. In May 1836, John Carter at age 21 had fallen from a tree. There was perfect absence of muscular power and sensibility of the skin throughout the body except in the head and upper part. In later years, John Hilton found indistinct sensibility on the left side as far as the elbow, ... muscles of the left shoulder were more developed than the right. The thumb was turned into the palm and the fingers were bent over.' (Sir Arthur Keith (1919) John Hilton. Principles of treatment. In: Menders of the Maimed. Oxford University Press.)

This astute observation by the esteemed nineteenth century orthopaedic surgeon, John Hilton (1807-1878), exemplifies not only the then-existing, but also the current problem of the flexion deformities of the

Correspondence: 95 Montgomery Drive Suite 114, Santa Rosa, CA 95405, USA. hand which not infrequently accompany traumatic cervical myelopathy. The presence of flexor hyperreflexia and hypertonicity, caudal to the injured cervical segment, is still often overlooked. In the presence of severe hyperreflexia, the appropriate measures of exercise and splinting have sometimes failed to prevent the subsequent development of fibrotic contracture in the flexor groups. In this series of 65 patients with spastic tetraplegic hands, the appropriate conservative methods had indeed failed to prevent the development of a functionally useless flexion posture of the hand with its consequent negation of the still intact wrist dorsiflexors' capacity to produce a tenodesis action. The hands described in this report were essentially 'club-like' and had little functional use to the patient.

A general agreement of the 1984 Giens Conference $^{1}$ (Table I) was that severe spasticity was a contraindication to surgery. A more recent report on some 300 muscle release operations for spasticity and spinal cord injury ${ }^{2}$ makes no reference to any procedures on the spastic hand. Faced with such stern interdiction, it at first appeared 
Table I International classification of the upper limb in quadriplegia (Giens, 1984)

\begin{tabular}{ll}
\multicolumn{2}{l}{ Motor grouping } \\
0 & $\begin{array}{l}\text { No muscle below elbow suitable for } \\
\text { transfer }\end{array}$ \\
1 & Brachioradialis (BR) grade 4 at least \\
2 & $\begin{array}{l}\text { Extensor carpi radialis longus (ECRL) plus } \\
\text { above }\end{array}$ \\
3 & Extensor carpi radialis brevis (ECRB) plus \\
& above \\
4 & Pronator teres (PT) plus above \\
5 & Flexor carpi radialis (FCR) plus above \\
6 & Finger extensors plus above \\
7 & Thumb extensors plus above \\
8 & Digital flexors plus above \\
9 & Lacks intrinsics only \\
$\mathrm{X}$ & Exceptions
\end{tabular}

Sensory grouping

' $\mathrm{O}$ ' if 2 point discrimination is greater than

$10 \mathrm{~mm}$ in the thumb

' $\mathrm{Cu}$ ' if 2 point discrimination is less than $10 \mathrm{~mm}$ in the thumb

(From McDowell et al $)^{1}$

foolhardy to employ the standard tendon transfers to this type of dyskinesia. Prior to the initial report, ${ }^{3}$ a similar attitude of pessimism had prevailed regarding any surgical approach to comparable dyskinesias in the hemiplegic hand, resulting from stroke or brain injury. Since 1961 significant improvement in the manual function of hemiparetic patients, following relatively simple tendon procedures, has been demonstrated by many authors. ${ }^{4,5}$ The increasingly successful employment of kinetic surgery, in patients capable of cooperating with the surgeon, suggested a possible application of these same procedures to the abnormal postures of the tetraplegic hand.

In 1964, a patient with severe flexion spasticity and contracture in both hands, resulting from a C6 traumatic myelopathy, urged that something be done about his flexed contractured fingers and thumb. Following selective elongation of the flexor tendons, he became able to use the operated hand as a very effective 'hook mechanism' to the extent that he later became a commercial photographer. The increased passive extension of the digits provided not only a functional grasp-prehension for small objects, but a more normal ability to approach and grasp larger objects. He became a most valuable member of our team in his capacity to demonstrate his improved manual function to later prospective patients. From this singular experience, it was felt that the prevailing pessimism about the surgical treatment of the spastic and contractured tetraplegic hand might indeed not be justified.

Initially, the aim was partly cosmetic, designed to modify the unwanted appearance of the hand, totally deprived of normal contact. ${ }^{8}$ When it was noted that a tenodesis action could be improved as a result of flexor tendon elongation at the wrist, it seemed appropriate to employ these techniques in the more demanding task of improving manual function. By careful intraoperative assessment of the appropriate length for each tendon, it became possible to provide an improved tenodesis function through active motion at the wrist. This method of merely 'softening' spasticity or contractures thereby avoided the inherent dangers of hypermobility and/or subsequent joint instability. ${ }^{7}$ An added advantage, following tendon lengthening of the digital flexors was the increased excursion of wrist dorsiflexion, resulting from surgical reduction of the preoperative 'tethering action' of the contractured digital flexors.

As the innervated 'motors' supplied by the preserved $\mathrm{C} 6$ and $\mathrm{C} 7$ radicles were not affected by these procedures, these intact tendons could then be utilized for appropriate transfers. Discussion of these tendon transfers, and of the patients who had some degree of preservation of the $\mathrm{C} 8$ nerve root supply, was not considered to be germane to this study, as these surgical techniques have been so completely described in historical reviews. ${ }^{8-10}$ The classification of preserved motor function, employed in almost all patients, was that established at the Giens meeting in 1984.

\section{Patients and methods}

As these procedures were initially viewed as being largely experimental, the more useless hand was chosen for surgery, but with 
increasing experience, the hand preferred by the patient was chosen. In the years 1964 through 1985, 65 tetraplegic patients underwent corrective operative procedures for reduction of their hyperreflexia and/or contracture. Fifty-two of these patients were male and 13 were female. The ages ranged between 18 and 44 years. All but 10 were involved in motor vehicle accidents, 6 patients had sustained diving injuries, 2 had gunshot wounds to the neck, and 2 resulted from benign resected cord tumors. The average interval between injury and surgery was 4.7 years.

Thirty-five patients were classified as having retained motor functions to the level of the C6 myotome (Giens classification IV). These patients had MRC ratings of $4 / 5$ at least in the brachioradialis $(\mathrm{Br})$, extensors carpi radialis longus and brevis (ECRL and ECRB), and in the pronator teres (PT). Thirty patients fitted in the Giens VI group, with the addition to the above muscles of the extensor carpi ulnaris (ECU) and extensor digitorum communis (EDC). All 65 patients had a combination of digital flexor spasticity and contracture and, owing to the late referral pattern, flexion contracture had largely replaced spasticity. Four of the more severely contractured hands had developed excoriation of the palmar skin and one had developed a palmar space infection.

A subgroup of 10 patients had an accompanying head injury. Preoperative evaluation of residual function in these hands was a difficult task. Surprisingly, after surgical elongation of the tight digital flexors, an unexpected degree of occult volitional extension of the digits, below the clinicallyassigned injured segment, was observed in 6 of these patients. Four of these 6 patients had dystonic ('intrinsic-plus') posturing at the metacarpophalangeal joints (MCP) of the fingers, which required additional surgical correction.

Preoperative sensory evaluation was performed according to the most widely acceptable technique. ${ }^{11}$ Patients were not accepted for this study unless they had adequate retention of sensation in the thumb and index. All patients had been provided with a full regimen of exercises and splinting during their initial hospital treatment.
Unfortunately, many of them had been remiss in continuing these programs in subsequent years. A fresh attempt at kinetic and static splinting, made prior to surgery for 2 additional months, had little benefit in terms of reducing contracture.

All procedures were performed using $0.5 \%$ local infiltration anesthesia without added vasoconstriction. This was done as it was soon apparent that the patient's active cooperation was a vital part in procuring the desired degree of tendon lengthening. The procedures were performed by 7 different surgeons, all of whom used a common protocol designed by the authors. As one of the author's (WJT) experience had been limited to the results of surgery in the spastic and dystonic hemiplegic hand, and as he also suffered the disadvantge of not being a surgeon, the cooperation and advice of the other authors was sought and generously provided throughout this study.

\section{Operative procedures}

\section{The hyperflexed digital posture (Giens IV patients)}

Employing local anesthesia, usually without the use of a tourniquet, the superficialis and profundus flexors (FDS and FDP) and the tendon of the flexor pollicis longus (FPL) were exposed by a curvilinear incision on the volar aspect of the wrist. The transverse carpal ligament was released in most patients to provide wider exposure of the flexor tendons. Local anesthesia was applied to the individual tendons, prior to their elongation by Z-plasty. The FDS tendons were then elongated and temporary sutures were applied to these. An intraoperative flexor reflex was performed on each of the individual digital flexors, to determine if sufficient reduction of hyperreflexia had been secured. The operated hand was then exposed to the patient's view, and he was asked actively to dorsiflex the wrist. If hyperreflexia, or contracture, was still present in the FDP tendons, these were then elongated, temporary sutures were applied, and the patient was asked actively to dorsiflex the wrist. Where indicated, the FPL tendon was similarly elongated to provide a 
more functional 'post' posture of the thumb.

Figure 1 shows the difference in extension range of the digits on the unoperated left hand, and the operated right hand, in 2 positions of the wrist, namely: (1) neutral wrist posture, and (2) with the wrist in slight palmar flexion. Some 25 years after the operation on the right hand, the patient had retained the original degree of tendon elongation and was able to use the provided increase in palmar surface to approach and grasp objects. Although a Giens IV patient, he became able postoperatively to develop complete independence in transfers, in feeding, self dressing, even in self catheterization, after the addition of a brachioradialis reinforcement to the FPL.

Due to the greater tendency of the ring and little finger flexors to develop recurrent contractures and their lack of proprioception, these tendons were more widely elongated than those of the other digits. The purpose of this was to ensure a wider surface of palmar contact, unobstructed by the later development of contractures in these digits. The provided increased extension was particularly appreciated by young adults in their manual approach to medium sized objects, such as a beer can, and their improved manual contact with their wheelchair rims. In 10 patients, additional minor surgical measures were needed to reduce capsular or ligamentous tightness of the IP or MCP joints, especially in those patients who had subluxed flexed joints. Four patients required intrinsic releases ${ }^{12}$ to reduce intrinsic contractures.

\section{The hyperflexed thumb posture}

Particular attention was paid to the precise degree of elongation needed in the FPL, in order to provide adequate tension, either for pulp apposition or 'key pinch'. The benefit of elongation of the FPL is shown in Figure 2. Following elongation of the FDS and the FDP, the hyperreflexic and contractured FPL denied this Giens IV tetraplegic patient an effective thumb-digital pinch (2a). After selective elongation of the FPL, he obtained wider palmar contact $(2 b)$, as well as good pulp contact between the thumb and index (2c). The contrasting posture of the unoperated left hand is clearly demonstrated in Figure 2(d).

\section{'Extrinsic-plus' posture (Giens VI group)}

In 18 patients with tightly flexed IP joints, in association with strongly contracting and inadequately antagonized EDC, flexor tendon elongation needed to be supplemented by measures to reduce the hyperextension posture of the MCP joints. An earlier use of dorsal capsulotomies was soon abandoned, as the resultant MCP flexion impeded the passive excursion of the digits in producing an effective tenodesis action. The 'lasso' procedure $^{13}$ was performed in 7 patients, with early excellent alignment and function after 6 months which was not sustained in later evaluations at one year. A persistent strongly contracting EDC in all but 2 patients caused recurrence of the 'extrinsic-plus' posture. In the remaining 5 of these patients, and in 11 other patients, the EDC was elongated under local anesthesia, proximal to the division of the common extensor tendons. Two patients required additional elongation of the EIP and ED $\mathrm{V}$ tendons. These procedures were supplemented by 'pinning' of the MCP joints in 30 degrees of palmar flexion for a period of 6 weeks postoperatively.

Shown in Figure 3 are the pre and postoperative appearances of the operated hand of a Giens VI patient who underwent surgery some 6 years after injury. Despite a rating of $4 \mathrm{MRC}$ power in the radial and ulnar wrist dorsiflexors, he was unable effectively to avail of these muscles because of the contractured finger and thumb flexors (Fig 3a and b). Following elongation of the digital flexors, the reflex overactivity of the EDC became apparent and he required a later elongation of this tendon. Postoperatively, he was provided with a much wider approach to objects during passive wrist palmar flexion (5c) and developed a strong pulp pinch with active wrist dorsiflexion (5d).

\section{Postoperative management}

A well padded cast was applied to the forearm and hand, extending distally to just 


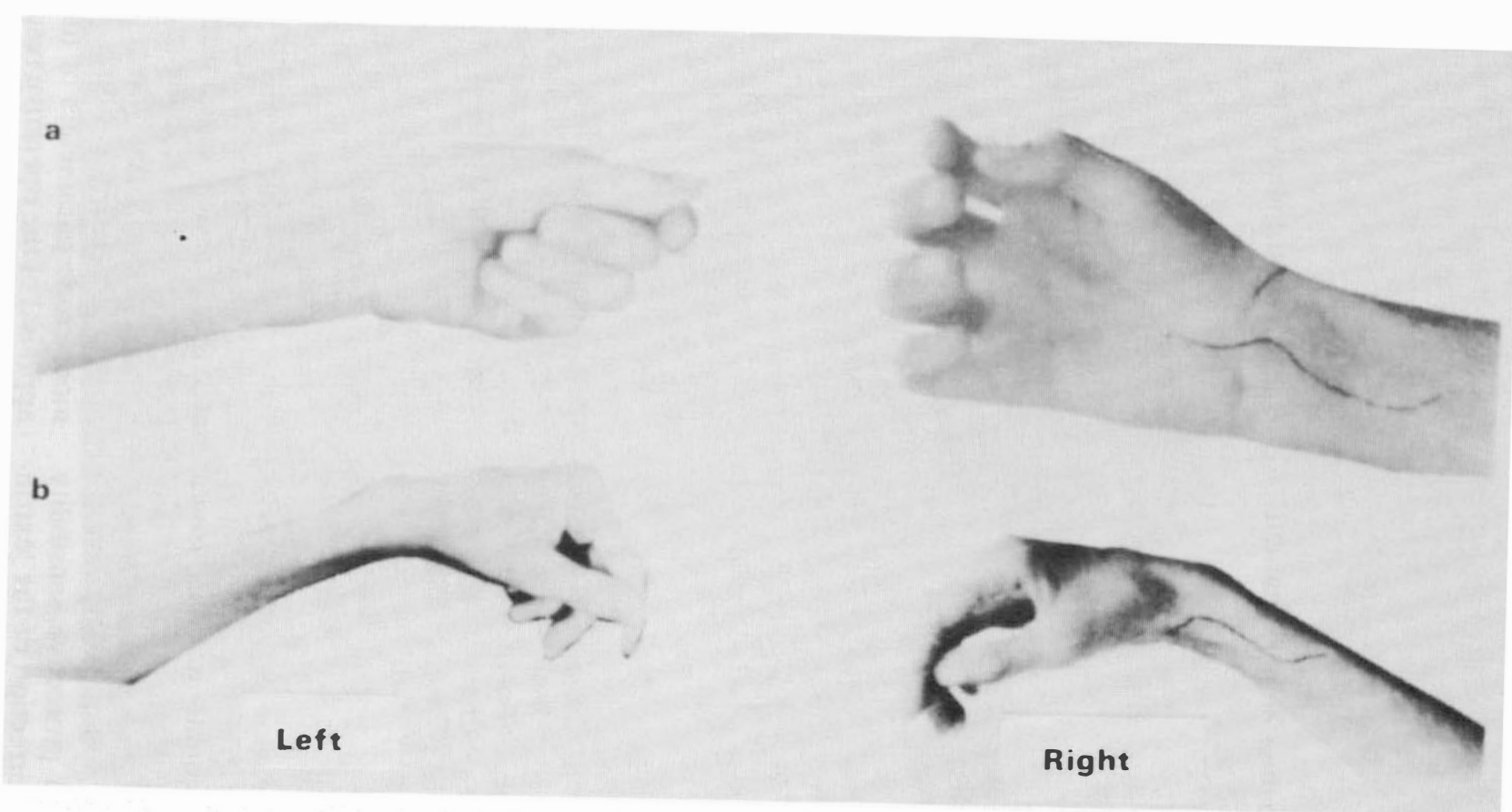

Figure 1 Contrasting postures of unoperated left hand, with operated right hand (25 years postoperative). 


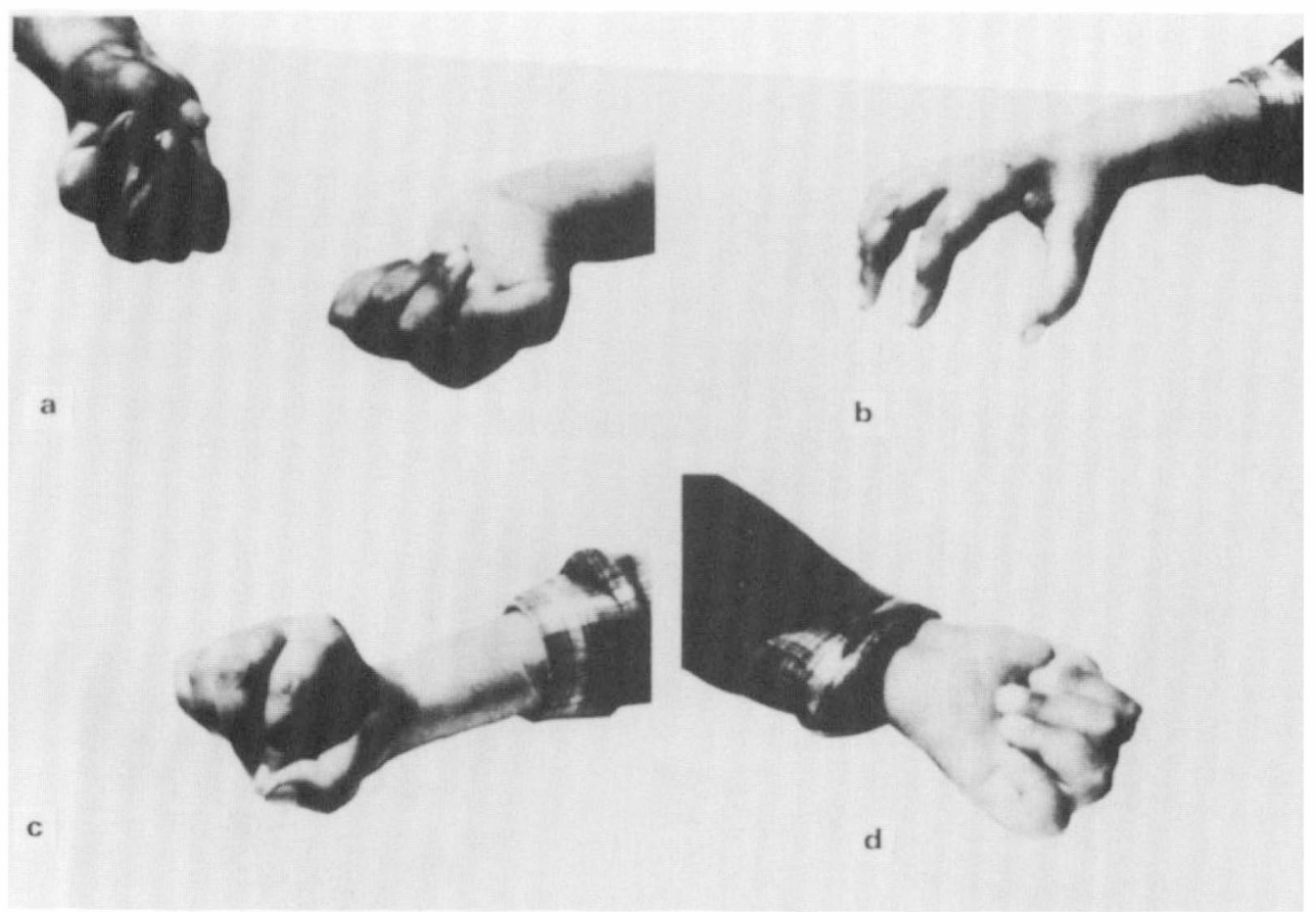

Figure 2 (a) Following inadequate elongation of FPL of operated right hand. (b) Following FPL elongation. (c) Functional tenodesis action with strong lateral pinch. (d) Unchanged posture of unoperated left hand.

beyond the terminal IP joints. To protect the lengthened flexor tendons, the cast was positioned at 15 degrees of palmar flexion at the wrist, with the thumb in a slightly abducted posture. The cast was removed after 3 weeks, and the patient was encouraged to perform active dorsiflexion of the wrist under the guidance of an occupational therapist.

If insufficient extension of the IP joints was obtained, small 'ring' splints (Fig 4a) were employed to provide increased digital extension range. The ease in adjustment and removal of these light splints allowed 7 patients to use them permanently, even in digits with impaired protective sensibility. Following surgical correction of the "extrinsic-plus' posture of the MCP joint, a dorsal metacarpal splint (Fig 4b) was applied for 3 months postoperatively to reduce recurrent hyperextension. The patient was then progressed through a therapy routine of devel- oping a pinching action with the use of varying sized objects, always being admonished to supplement proprioceptive relays by the addition of visual cues.

\section{Operative results}

It was not feasible to use the refined methods of functional evaluation as outlined in an earlier paper $^{14}$ to determine the efficacy of these procedures, as preoperatively these patients had such a low level of motor performance. Instead, the postoperative assessment had to depend on the more crude parameters of the patient's self appraisal of the operative results, and on the objective evaluations performed by the surgeon. ${ }^{15}$ These clinical observations were also complemented by activities of daily living (ADL) assessments performed by occupational therapists. Postoperative re- 


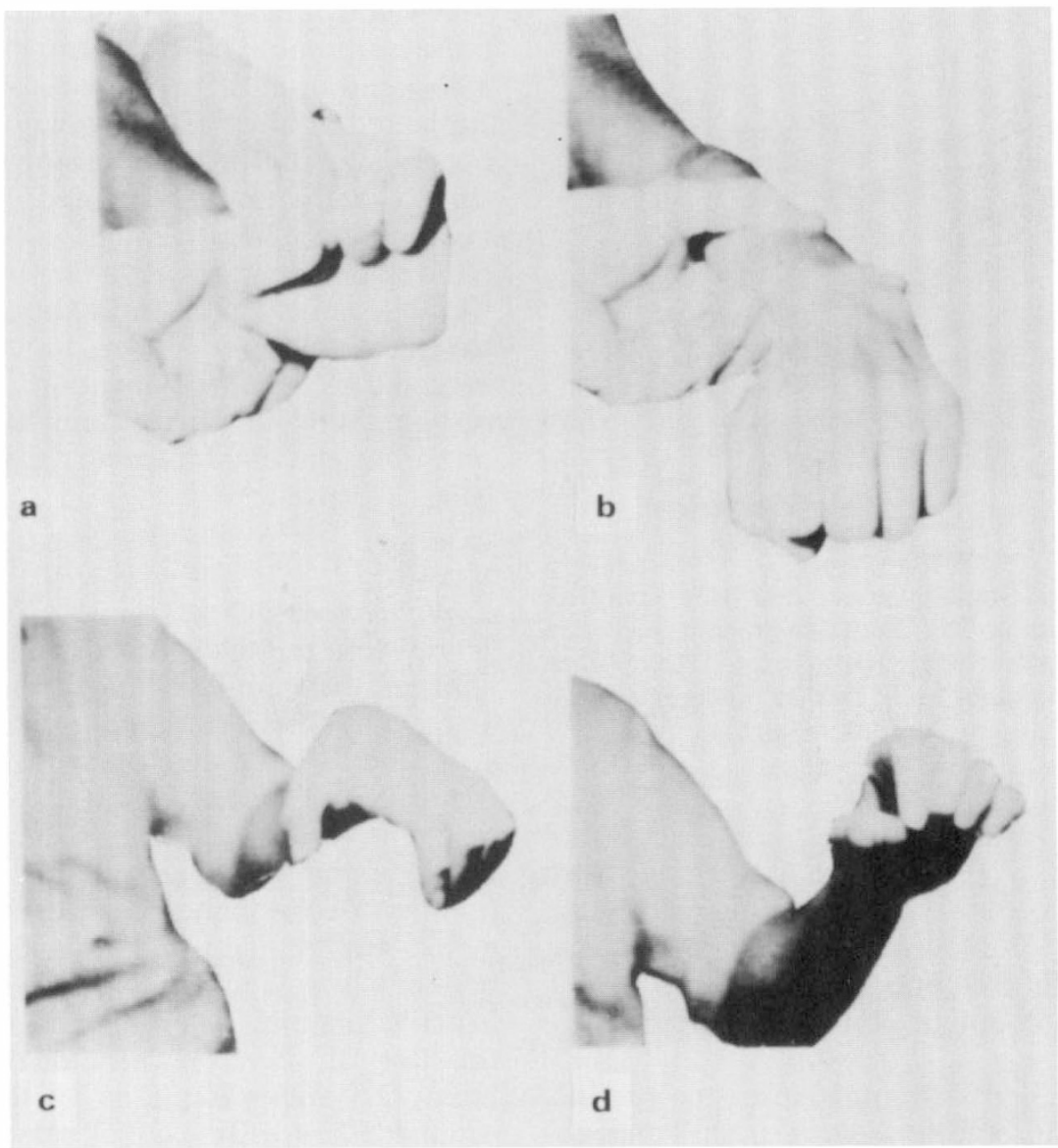

Figure 3 (a) Preoperative dysfunctional tenodesis action of left hand due to contractured thumb and finger flexors. (b) Flexion contractures not reduced with wrist palmar-flexed. (c) Postoperative elongation of FDS, FDP, and EDC. (d) Postoperative tenodesis action.

sults were determined at 6-, 12-, and 24-month intervals in all but 9 patients. These 9 patients were lost to follow up at the end of one year.

\section{Improved manual contact}

That this was improved in all patients in both groups was not a surprising result, as palmar contact was substituted for the previous contact solely along the dorsal surface of the hand. The reduction of contracture was particularly appreciated by the patients not only because of the improved cosmetic appearance, but also for their ability to make more normal manual contact with their colleagues and partners. Preoperatively these hands could be employed only as a 'pushing member', without any capacity for grasp or release. With surgical 'softening' of the hyperreflexia and contracture by tendon lengthening, all but 2 patients were provided with an effective tenodesis action. These 2 patients showed inadequate reduction of flexor hyperreflexia, and needed repeat elongations.

\section{Grasp-prehension function}

Postoperatively a 'key pinch' mechanism was utilized in most patients. In 2 patients, the FPL had been too widely elongated, 


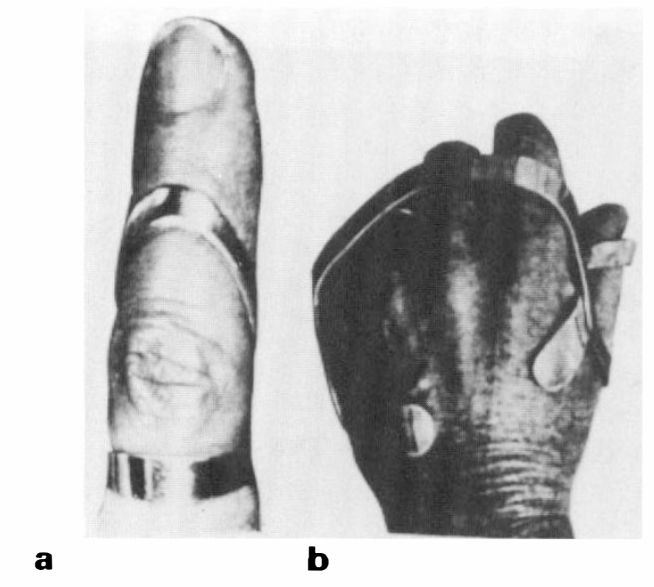

Figure 4 (a) Static postoperative 'ring' splint for stabilization of PIP joint to prevent recurrent flexion contracture. (b) Postoperative static splint to maintain MCP flexion posture.

with the resultant weak 'pulp-pinch', and these hands required reinforcement with tendon transfers. Otherwise, overcorrection was not a problem in these patients, due to the careful retention, at surgery, of the desired amount of contracture to stabilize the joints.

In the Giens VI hands, after the production of appropriate flexor tendon lengthening, a persistent extension posture of the MCP joints significantly impaired the tenodesis action of 18 out of the 30 hands. Two were successfully corrected by the Zancolli procedure. In the remaining 16 patients, elongation of the 'extrinsic extensor' tendons successfully corrected this posture at 2 years follow up. Two patients needed repeat elongation of the EDC. Elongation of this tendon was of especial benefit in 4 patients, in whom preoperative clonus of this muscle had been a particularly disabling feature. In the remaining 12 patients, MCP hyperextension was of a relatively minor order, and could be easily corrected by a dorsal metacarpal splint. The overall functional results obtained in this group, as expected, were much greater than those obtained in the Giens IV subset. This was due largely to the better existing balance between the radial and ulnar dorsiflexors of the wrist and the wider area of digital sensibility.
A wholly unexpected discovery of 'occult' preserved motor function in 6 patients with associated brain injury could not be readily explained on the basis of any possible 'root escape', as the pre and postoperative sensory examinations remained unchanged. Postoperative improvement in function implied rather that the prevailing preoperative flexor spasticity and contracture had denied the patients these hidden functions, later revealed by tendon elongation. An analogous phenomenon of deafferentation's effect on suprasegmental recovery has been recently reported. ${ }^{16}$

\section{Complications}

These were relatively few in number, in the light of the simplicity of the procedures. Three patients developed a carpal tunnel syndrome 3 to 6 months postoperatively, requiring release of the transverse carpal ligament. In subsequent patients, release of this ligament was routinely employed. The finding of pseudoneuromata in the median nerve was thought to be caused by the patient's increased use of the operated hand in activating their wheelchairs. In 2 patients, the use of a vasoconstrictor resulted in temporary hypovascularity of the mediansupplied digits. By careful removal of the median nerve from the infiltrated field, and the discontinuance of a vasoconstrictor, this complication was subsequently avoided. The potential for fluid overload from intravenous solution was overlooked in 3 of our older patients. Preoperatively, these patients had a delicate reflex bladder-voiding mechanism, and developed postoperative distension of the bladder. Prophylactically, all patients were then operated with an indwelling catheter, or had prompt catheterization after surgery.

In 3 patients, deterioration of the provided functional improvement was noted approximately one year postoperatively. They were found by MRI to have a posttraumatic syringomyelia unrecognized prior to surgery. With cephalad extension of the syrinx, they had developed 'spasms' in either the forearm flexors or extensors, and these patients subsequently needed syringostomies to prevent further functional loss. 
This discovery prompted the employment of preoperative MRI evaluation in all patients, especially in those in whom reflex responses, below the level of segmental injury, were found to be hypoactive or "paradoxical'. This finding would suggest that patients with irradiating 'spasms of the upper extremities' should not undergo reconstructive surgery until an MRI had been obtained.

\section{Discussion}

While many authors have viewed severe spasticity as an absolute contraindication for the employment of corrective surgery, especially of tendon transfers, the results obtained in this study would suggest that this prevailing pessimism can be lightened by the described minor procedures. In a previous report, the alternatives of phenol and alcohol nerve blocks have been found to pose hazards of causalgia and unpredictability. ${ }^{17}$ Tenotomies, except for cosmesis in severely contractured hands, are of little proven functional value. Release of the origins of the spastic flexor muscles at the elbow has previously been reported ${ }^{18}$ to be inferior functionally to their elongation at the wrist. Indeed, the attendant complications from wide dissection at the elbow, resulting in a dysfunctional supinated posture of the forearm, have been cogently outlined by the Rancho Los Amigos group. ${ }^{19}$

While the 'worst possible scenario' of a tightly flexed hand was the basis of this study, the functional results obtained would suggest that these measures would have had even more beneficial application at an earlier stage, before the evolution of spasticity into fibrotic contracture. The departure from the more customary use of a tourniquet was necessitated in this series by the need to maintain proprioceptive sensation throughout the operation. Without the use of a tourniquet, the extra operative time was no more than 10 minutes.

While elongation of the digital extensor tendons was employed in only 16 patients, the functional results obtained would suggest that this simple procedure is a useful addition in the treatment of dysfunctional 'extrinsic-plus' posture, found in some patients with IP flexor spasticity and/or contracture. These results suggest that these particular kinetic disorders are perhaps better redressed by the described kinetic means in a wide-awake patient. The provided reduction of spasticity and contracture, and the improved architectural posture of the hand, also allowed for the later employment of tendon transfers. Certainly, not one of the patients in this series would have derived any functional benefit from tendon transfers alone, without prior redressment of their spasticity or contracture. These procedures offer the additional safeguard in their easy reversibility if the results should fail to meet the patient's functional requirements.

Although this report appears to emphasize the mechanical benefits provided, it should not be interpreted as a departure from an earlier emphasis on the eminent need for improving afferent input to the spinal synapse. By selective tendon lengthening, the wider hand posture provided increased afferent return from the volar surfaces of those digits which had intact sensibility. This concept of modifying the central 'keyboard' has been aptly described by Gooddy ${ }^{20}$ in his article on sensation and volition, as follows: 'In voluntary movement, sensation and motion form a single and indivisible continuous process'.

\section{Acknowledgements}

This study was made possible through the generous cooperation of 2 esteemed deceased orthopedic colleagues, Ernst Dehne and Lloyd Taylor, whose pioneer efforts were later refined by Drs Garnet Wynne, Michael Sommer, William Teipner, James Bauer, and Frederic Born.

\section{References}

1 McDowell CL, Moberg EA, House JH (1986) The second international conference on surgical rehabilitation of the upper limb in tetraplegia (quadriplegia). J Hand Surg 11A: 604-608.

2 Eltorai I, Montroy R (1990) Muscle release in the management of spasticity in spinal cord injury. Paraplegia 28: 433-440. 
3 Treanor WJ, Reifenstein GH (1961) Potential reversibility of the hemiplegic posture: results of reconstructive surgical procedures. Am J Cardiol 7: 370-378.

4 Waters RL, Wilson DJ, Hecker RS (1984) Rehabilitation of the upper extremity after stroke. Rehabilitation of the Hand. 2nd ed., CV Mosby Co, St Louis: 705-715.

5 Roper BA (1987) The orthopaedic management of the stroke patient. Clin Orthop 219: 78-86.

6 Moberg E (1975) Surgical treatment for absent single-hand grip and elbow extension in quadriplegia. $J$ Bone Joint Surg 57A: 196-206.

7 Moberg E (1987) The present state of surgical rehabilitation of the upper limb in tetraplegia. Paraplegia 25: $351-356$.

8 Moberg E (1978) The Upper Limb in Tetraplegia: A New Approach to Surgical Rehabilitation. Georg Thieme Publishers, Stuttgart.

9 Hentz VR, Keoshian LA (1979) Changing perspective in surgical hand rehabilitation in quadriplegic patients. Plast Reconstruct Surg 64: 509-515.

10 Johnstone BR, Jordan CJ, Buntine JA (1988) A review of surgical rehabilitation of the upper limb in quadriplegia. Paraplegia 26: 317-339.

11 Moberg E (1983) The role of cutaneous afferents in position sense, kinaesthesia and motor function of the hand. Brain 106: 1-19.

12 Smith RJ (1977) Surgery of the hand in cerebral palsy. Operative Surgery-The Hand. 3rd ed. Butterworths, London-Boston: 218-230.

13 Zancolli E (1975) Surgery for the quadriplegic hand with active strong wrist extension. Clin Orthop 112: 101-113.

14 Waters RL, Moore KR, Graboff WR, Paris K (1985) Brachioradialis to flexor pollicis longus tendon transfer for active lateral pinch in the tetraplegic. J Hand Surg 10A: 385-391.

15 Hentz VR, Brown N, Keoshian LA (1983) Upper limb reconstruction in quadriplegia. Functional assessment and proposed treatment modifications. J Hand Surg 8A: 119-131.

16 Little JW, Harris RM, Smithson D (1989) Motor recovery in the absence of segmental afferents. A case study of incomplete spinal cord injury. Paraplegia 27: 385-389.

17 Treanor WJ (1969) The hemiplegic posture and its correction. Clin Orthop 63: 113-131.

18 Treanor WJ (1981) Improvement of function in hemiplegia after orthopaedic surgery. Scand J Rehabil Med 131: $123-135$.

19 Keenan MA, Abrams RA, Garland DE, Waters RL (1987) Results of fractional lengthening of the finger flexors in adults with upper extremity spasticity. J Hand Surg 12A: 575-581.

20 Gooddy W (1949) Sensation and volition. Brain 72: 312-329. 